\title{
Fully Adaptive Power Saving Protocols for Ad Hoc Networks Using the Hyper Quorum System
}

\author{
Shan-Hung Wu ${ }^{\ddagger \dagger \downarrow}$, Ming-Syan Chen ${ }^{\ddagger}$, and Chung-Min Chen ${ }^{\dagger}$ \\ ${ }^{\ddagger}$ Department of Electrical Engineering, National Taiwan University, Taipei, Taiwan, ROC \\ ${ }^{\dagger}$ Telcordia Technologies, Piscataway, NJ, USA \\ ${ }^{\natural}$ Industrial Technology Research Institute, Hsinchu, Taiwan, ROC \\ \{brandonwu, chungmin\}@research.telcordia.com,mschen@cc.ee.ntu.edu.tw
}

\begin{abstract}
Quorum-based Power Saving (QPS) protocols have been proposed for ad hoc networks (e.g., IEEE 802.11 ad hoc mode) to increase energy efficiency and prolong the operational time of mobile stations. These protocols assign to each station a cycle pattern that specifies when the station should wake up (to transmit/receive data) and sleep (to save battery power). In all existing QPS protocols, the cycle length is either identical for all stations or is restricted to certain numbers (e.g. squares or primes). These restrictions on cycle length severely limit the practical use of QPS protocols as each individual station may want to select a cycle length that is best suited for its own need (in terms of remaining battery power, tolerable packet delay, and drop ratio). In this paper we propose the notion of Hyper Quorum System (HQS) - a generalization of QPS that allows for arbitrary cycle lengths. We describe algorithms to generate two different classes of HQS given any set of arbitrary cycle lengths as input. We then present analytical and simulation results that show the benefits of HQS-based power saving protocols over the existing QPS protocols.
\end{abstract}

\section{Introduction}

Ad hoc network technologies and standards, such as IEEE 802.11's ad hoc mode, allow the quick set-up of a wireless network among a group of mobile stations, where the stations communicate with each other either directly or indirectly through multiple hops, without the aid of an infrastructure (e.g., cables, access points or base stations). The mobile stations (devices) in the network usually rely on batteries as the power source, thus, ensuring energy efficiency during ad hoc communication is essential to prolonging the operational time of the devices.

Energy conservation could be achieved at different layers with different techniques. When a station is not transmitting, the transceiver at PHY layer persists in idle mode and continuously listens for incoming transmissions. Studies $[7,12]$ find that the energy consumed by a wireless module in listening to the network is only slightly lower than that of transmitting and receiving data. Therefore, if there are seldom transmissions destined for the station, idle listening would waste significant amount of energy. To address this problem, a power management policy is usually adopted at MAC layer which, instead of idle listening, allows a station to sleep (or doze) - to suspend the transceiver - when there is no data transmission.

The IEEE 802.11 Power-Saving (PS) mode [6] includes a mechanism that allows a station to go into sleep when there is no data transmission. The standard divides the timeline evenly into beacon intervals and requires all stations to wake up at the beginning of each beacon interval to check if there is data transmission request from other stations (for this purpose all stations must have their clocks synchronized). A station will goes into sleep for the rest of a beacon interval if there is no data transmission request from other stations destined for the station. Nonetheless, studies [9, 20] have shown that the IEEE 802.11 PS mode is not energy efficient under light data traffic loads, because a station still needs to remain awake for a short time window at the beginning of each beacon interval. To address this problem, recent work [8, 16, 19] has explored the Quorum-based Power Saving (QPS) protocols which require a station to wake up only for certain beacon intervals (instead of all beacon intervals as in 802.11 PS mode). Given an integer $n$, a quorum system defines, for each station in the network, a cycle pattern (or cycle for short) that specifies the awake/sleep schedule for $n$ continuous beacon intervals. This pattern repeats every $n$ beacon intervals and therefore $n$ is called the cycle length. The QPS protocols require a station to remain awake for only $O(\sqrt{n})$ beacon intervals per cycle and guarantees 
that any two stations will overlap in at least one awakened beacon interval within each cycle. A QPS system also ensures that the collective awakened beacon intervals from all stations are distributed uniformly within a cycle so that the bandwidth of the network can be fully utilized. It has been shown that QPS protocols render much better power saving than IEEE 802.11 PS mode under light traffic loads.

In QPS protocols, there is a trade-off when it comes to selecting a value for the cycle length $n$ - the larger the value of $n$, the more the power saving; yet the longer the buffering delay at the sender as data must be buffered until the recipient wakes up. Requiring all stations to use the same value of cycle length is problematic as individual stations may have their own delay requirement on data communications and constraint of remaining battery power. This consideration leads to a number of recent work $[4,5]$ that allow the stations to use different values of cycle length $n$, with certain restrictions. In AQEC [4] $n$ is required to be a square; while in AAPM [5] $n$ must be a prime number. We call these QPS systems semi-adaptive in the sense that they allow stations to use different cycle lengths but only with certain values. In reality, both these systems render a very limited selection for the values of $n$, as the cycle length must be small (typically, $n \leq 25$ ) due to the consideration of node mobility and/or route advertisement interval at the network layer [15]. Studies $[2,11,21]$ have shown that devices can end up consuming excessive energy if any inappropriate cycle length is used. This urges the need for a new power management solution that offers more flexibility in the selection of cycle lengths.

In this paper, we propose and define the notion of Hyper Quorum System (HQS) that generalizes traditional quorumbased systems to allow for arbitrary cycle lengths for different mobile stations, while guaranteeing overlap of awakened periods between any two stations. We note that HQS is "fully adaptive" in the sense that a station can select any value of cycle length that is best suited to its own requirements in terms of packet delay and power constraint. We propose two constructive algorithms to build HQS schemes, one based on the semi-adaptive grid QPS [16] and another based on the difference set [14], in $O(1)$ time. To the best of our knowledge, these are the first known fully adaptive quorum-based power management protocols for ad hoc networks. Experimental results show that the HQS-based power management protocol may offer significantly better performance than traditional QPS protocols. In particular, it yields up to $41 \%$ improvement in energy efficiency under heavy traffic loads.

The rest of this paper is organized as follows. In Section 2 , we review existing power management protocols for ad hoc networks. Section 3 formally defines the notion of HQS and describes two HQS constructing schemes. The power management protocol based on HQS is also described. Sec- tion 4 presents the performance evaluation of our protocols based on both analytical and simulation results. Section 5 concludes the paper.

\section{Preliminaries}

In this section, we review existing power management protocols for ad hoc networks.

\subsection{IEEE Power Saving Mode}

IEEE 802.11 [6] is currently one of the most popular MAC standard for wireless ad hoc networks. The operation of IEEE 802.11 Power Saving (PS) mode is shown in Figure 1(a). The time axis is divided evenly into beacon intervals and all stations are synchronized to align on these intervals. At the beginning of each beacon interval is an ATIM (Announcement Traffic Indication Message) window. Each station is required to wake up and remain awake during the entire ATIM window.

If a station, say $H_{1}$, intends to transmit data to a destination, say $H_{0}$ (Figure $1(\mathrm{a})(1)$ ), it first unicasts an ATIM frame to $H_{0}$ during the ATIM window (Figure $\left.1(\mathrm{a})(2)\right) . H_{0}$, upon receiving the ATIM frame, will send back an acknowledgement. After this ATIM notification procedure, both $H_{0}$ and $H_{1}$ will keep awake for the rest of the beacon interval, during which the DCF (Distributed Coordination Function) mechanism (i.e., RTS, CTS, and random back-off) [6] will be initiated to transmit the data (Figure 1(a)(3)) while avoiding collisions ${ }^{1}$.

If a station neither sends out nor receives an ATIM notification during the ATIM window, it may enter the doze mode (that is, to sleep) for the rest of the beacon interval.

In IEEE 802.11 PS mode, the Target Beacon Transmission Time (TBTT) on all stations should be aligned to ensure the overlap of ATIM windows. To synchronize the timer, all stations contend to send a beacon frame, which carries timer information, at the beginning of a beacon interval. Upon hearing the first beacon, each station synchronizes its clock with the carried information and cancels its own beacon transmission.

\subsection{Quorum-based Power Saving Proto- cols}

Existing Quorum-based Power Saving (QPS) protocols for ad hoc networks can be classified into two categories: the static and semi-adaptive protocols. The static QPS protocols, which require all stations to use the same cycle length, can be further divided into two sub-categories:

\footnotetext{
${ }^{1}$ Note in the situation where data transmission cannot complete within a single beacon interval (due to collisions or large data volume), $H_{1}$ can set the more-data bit (in frame header) true telling $H_{0}$ to remain awake through the successive beacon interval, and then the data transmission continues [6].
} 

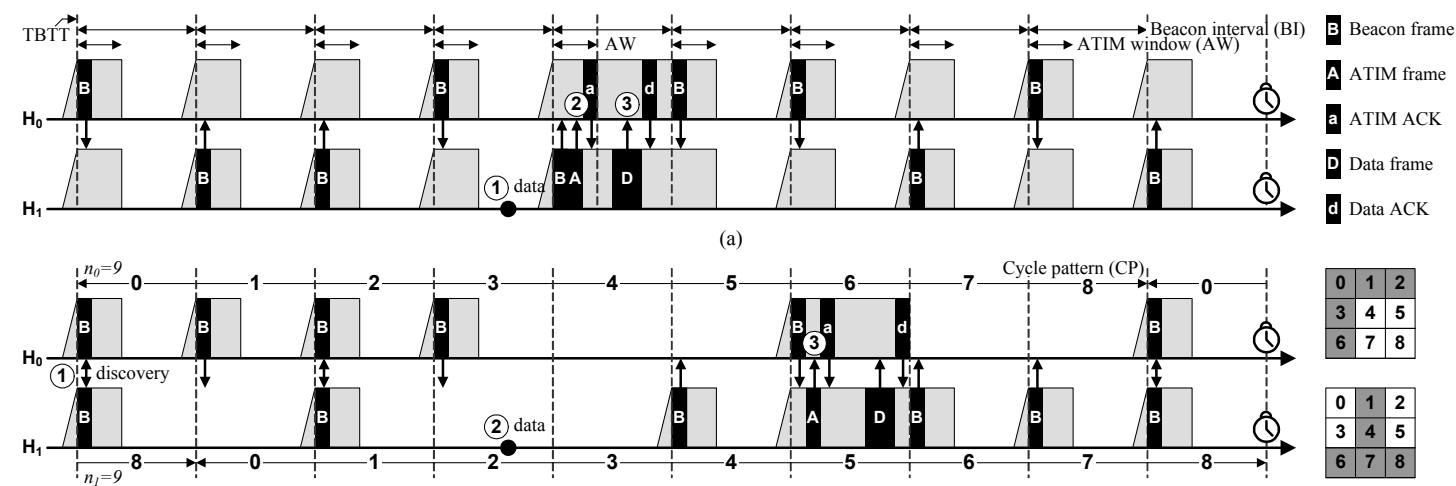

(b)

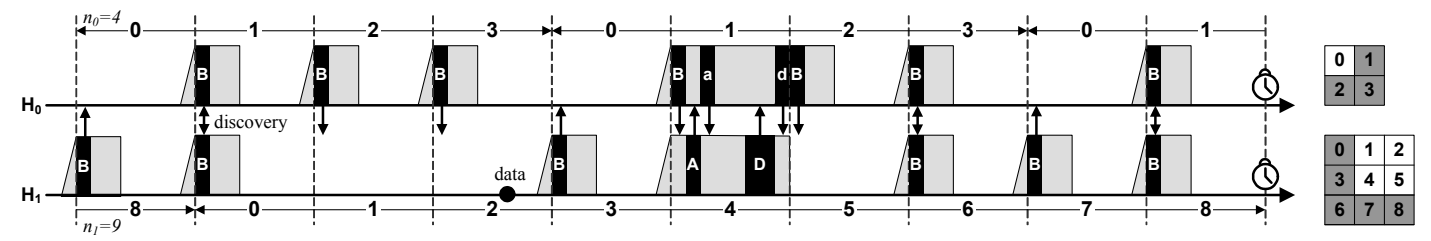

(c)

Figure 1. Existing ad hoc power management protocols. (a) IEEE Power Saving (PS) mode. (b) Static grid/torusbased quorum systems. (c) AQEC with semi-adaptivity.

synchronous and asynchronous. Synchronous QPS [16, 17] rely on a timer synchronization mechanism to ensure the overlap of awakened periods between each pair of stations. The asynchronous QPS protocols [8, 19] ensure the overlap of awakened periods even when there is no timer synchronization mechanism. The latter protocols are more scalable to large numbers of stations as timer synchronization is costly in such situations. This advantage however comes at the expense of less energy efficiency as a station will need to stay awake for the entirety of a beacon interval for which the station is scheduled to wake up (in contrast, in synchronous QPS a station only needs to stay awake during the ATIM windows of those beacon intervals). Recently, the semi-adaptive QPS protocols, AQEC [4] and AAPM [5], were proposed that allow stations to choose different cycle lengths with certain restrictions on the values that can be selected from.

In the following we briefly summarize the static, gridbased QPS protocols $[8,16]$ and the semi-adaptive AQEC protocol [4] as they are relevant to our study.

Grid-based QPS protocols: Figure 1(b) illustrates the awake/sleep schedules of two stations, $H_{0}$ and $H_{1}$, which are defined by a grid quorum scheme with cycle length $n=9$. The grid quorum scheme organizes every $n$ continuous beacon intervals into an $\sqrt{n} \times \sqrt{n}$ array and numbers them from 0 to $n-1$ in a row-major manner. It defines a quorum as a set containing the numbers along an arbitrary row and an arbitrary column in the array (e.g., $\{0,1,2,3,6\}$ or $\{1,4,6,7,8\}$ as shaded in Figure 1(b)). Each station, by using this scheme, is able to obtain its own quorum of quorum size (i.e., cardinality) $2 \sqrt{n}-1$. For all beacon intervals whose numbers are specified in the quorum, the station will remain awake during the ATIM windows (as in IEEE 802.11 PS mode). For beacon intervals whose numbers do not appear in the quorum, the station will sleep without even awaking for the ATIM windows. Such a schedule repeats every $n$ beacon intervals and is called the cycle pattern (or cycle for short). We denote the duration of the ATIM window and beacon interval as $\bar{A}$ and $\bar{B}$ respectively. This scheme gives the lowest duty cycle (i.e., the portion of time a station remains awake) $\frac{(2 \sqrt{n}-1) \bar{A}}{n \bar{B}}$. Apparently, the larger the cycle length $n$, the more the power saving.

In QPS protocols, a beacon frame should carry additional information about the schedule, including the adopted quorum and the number of current beacon interval, etc. Unlike the IEEE 802.11 PS mode where a station should cancel its own beacon transmission upon hearing the first beacon frame, each station should persist its beacon transmission (even when the others' beacons are heard) to claim its own schedule.

As we can see in Figure 1(b), each quorum in the grid quorum scheme intersects with any other quorum in two elements. This implies that the ATIM windows between stations overlap twice per cycle. Once beacon frames are exchanged at an overlapped period (Figure 1(b)(1)), stations $H_{0}$ and $H_{1}$ are able to discover each other; that is, to re- 
ceive and keep one another's schedule, and to predict the next coming of ATIM window at the other party. Suppose $H_{1}$ has data for $H_{0}$ (Figure 1(b)(2)), it buffers the data and waits for the next ATIM window of $H_{0}$. When $H_{0}$ wakes up (Figure 1(b)(3)), $H_{1}$ unicasts an ATIM frame to $H_{0}$ and starts the notification and data transmission procedures as described previously in the IEEE 802.11 PS mode.

It is important to note that the grid quorum scheme ensures the overlaps of ATIM windows even when the numbering of beacon interval shifts between stations. For example, as we can see in Figure 1(b) $H_{0}$ 's clock leads $H_{1}$ 's clock by one beacon interval. The quorum adopted by $H_{0}$, from $H_{1}$ 's point of view, becomes $\{0-1(\bmod 9), 1-$ $1(\bmod 9), 2-1(\bmod 9), 3-1(\bmod 9), 6-1(\bmod 9)\}=$ $\{8,0,1,2,5\}$. We can easily verify that the rotated schedule of $H_{0}$ still overlaps twice per cycle with that of $H_{1}$. This nice property is due to the fact that the intersections between $H_{0}$ 's and $H_{1}$ 's quorums are shift-invariant ${ }^{2}$. Apparently, only those quorum schemes producing shift-invariant quorums can be used in a QPS protocol. The lower bound of the size of these shift-invariant quorums is $\sqrt{n}$ [8].

The power saving advantage provided by QPS protocols comes at the price of delay. Such delay includes the data buffering time, i.e., the duration between a packet arrival (on a sending station) and its start of DCF. As we can see in previous examples, the data buffering time in grid-based QPS protocol is at most $O(\sqrt{n}) \bar{B}$. Besides, two adjacent stations may not be able to discover each other until a cycle passes by. The neighbor discovery time, i.e., the time for a station to discover its new neighbor, is therefore $O(n) \bar{B}$ in the worst case.

Semi-adaptive AQEC protocol: The static, grid-based quorum scheme is extended to a semi-adaptive one in AQEC [4], where each station is free to choose a cycle length, given that it is a square number. Figure 1(c) illustrates an example where $H_{0}$ and $H_{1}$ adopt cycle patterns with different square lengths $n=4$ and $n^{\prime}=9$ respectively. It can be shown that $H_{0}$ and $H_{1}$ are guaranteed to receive one another's beacon frame at least once every $\sqrt{n_{\min }}+n_{\max }-1$ beacon intervals, where $n_{\min }=$ $\min \left\{n, n^{\prime}\right\}$ and $n_{\max }=\max \left\{n, n^{\prime}\right\}$. This implies that the neighbor discovery time is $O\left(\sqrt{n_{\min }}+n_{\max }-1\right) \bar{B}$.

Another scheme, which is based on the Finite Projective Plane (FPP) [5], gives similar flexibility except that $n$ must be a prime. The FPP-based scheme has one serious drawback as compared with the grid scheme-it does not give bounded neighbor discovery time. This makes FPP hard to be applied to the real networks, since stations are usually required to discover/update its neighbors once within a certain period (e.g., a route advertisement interval).

As mentioned earlier, requiring cycle lengths to be

\footnotetext{
${ }^{2} \mathrm{~A}$ quorum scheme that guarantees the shift-invariant intersection is called cyclic. We will formally define the cyclic property in Section 3.
}

squares or primes could be too much a stringent requirement as in practice the cycle lengths are usually smaller than 25 , due to the node mobility and routing requirement ${ }^{3}$. Studies $[2,11,21]$ have shown that a PS station compromising on a sub-optimal cycle length may end up consuming more energy than a non-PS station because of the excessive packet drops and re-transmissions. Apparently, to allow a fine-tunable cycle length on each station is a key to success for a QPS protocol.

\section{The Hyper Quorum System}

This section defines the notion of Hyper Quorum System (HQS) and describes algorithms to construct two different classes of HQS. HQS is a generalization of traditional quorum systems [3] where stations may obtain cycle patterns of arbitrary lengths that best suit their requirements.

The advantage offered by HQS is two-fold. First, HQS allows more energy saving on those stations having critical battery power or light traffic load. This overall prolongs the lifetime of a network. In addition, HQS gives controllable delay that is able to meet application requirements. This avoids serious performance degradation and energy waste on packet re-transmissions as induced by traditional power management protocols. Note this advantage becomes significant when all peers in a network have distinct requirements of energy consumption and delay, which is usually true due to the number of hops, battery life, mobility, and running applications.

\subsection{Definitions and Fundamentals}

Consider the sets in which each element denotes a number of beacon interval. The following definitions are based on $[3,14]$.

Definition 3.1 (n-coterie) Given an integer $n$ and $a$ universal set $U=\{0,1, \cdots, n-1\}$ over the modulo- $n$ plane. Let $X$ be a set of nonempty subsets of $U$. We call $X$ an $n$-coterie if and only if for all $Q, Q^{\prime} \in X, Q \cap Q^{\prime} \neq \varnothing$.

For example, the set $\{\{0,1,2,3,6\},\{1,4,6,7,8\}\}$ given in Figure 1(b) is a 9-coterie. Conventionally, a coterie $X$ is termed a quorum system, and the elements of $X$ (i.e., $Q$ ) are called the quorums.

Not every quorum system is applicable to a QPS protocol [8]. In a QPS protocol, two quorums must intersect even when one "shifts." This leads to the following definitions:

Definition $3.2((n, l)$-cyclic set) Given integers $n$ and $l$, where $0 \leq l \leq n-1$. Let $Q$ be a subset of $U, U=$

\footnotetext{
${ }^{3}$ In the simulation part of study [4], AQEC provides only 4 practical choices (i.e., 4, 9, 16, and 25) for cycle lengths.
} 


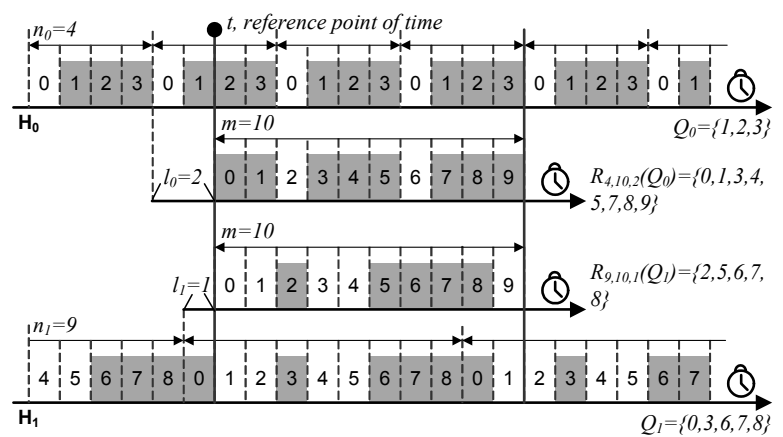

Figure 2. Rather than ensuring the intersection between quorums, the hyper quorum system guarantees the intersection between projections of quorums over a modulo- $m$ plane.

$\{0,1, \cdots, n-1\}$. We call $C_{n, l}(Q)$ an $(n, l)$-cyclic $\operatorname{set}$ of $Q$ if and only if $C_{n, l}(Q)=\{(q+l) \bmod n: \forall q \in Q\}$.

For convenience, we denote a group of cyclic set as $C_{n}(Q)=\left\{C_{n, l}(Q): \forall l\right\}$.

Definition 3.3 ( $n$-cyclic quorum system) Given an integer $n$ and $a$ universal set $U=\{0,1, \cdots, n-1\}$ over the modulo-n plane. Let $X=\left\{Q_{0}, Q_{1}, \cdots, Q_{d-1}\right\}, d \in \mathbb{Z}$, be a set of nonempty subsets of $U$. We call $X$ an $n$-cyclic quorum system if and only if the set $C_{n}\left(Q_{0}\right) \cup C_{n}\left(Q_{1}\right) \cup$ $\cdots \cup C_{n}\left(Q_{d-1}\right)$ is an n-coterie.

For example, the set $\{\{0,1,2,3,6\},\{1,4,6,7,8\}\}$ also forms a 9-cyclic quorum system, as every element pair in

$$
\begin{aligned}
& \{\{0,1,2,3,6\},\{1,2,3,4,7\}, \cdots,\{8,0,1,2,5\}\} \cup \\
& \{\{1,4,6,7,8\},\{2,5,7,8,0\}, \cdots,\{0,3,5,6,7\}\}
\end{aligned}
$$

intersects. The cyclic property ensures the shift-invariant intersection between $\{0,1,2,3,6\}$ and $\{1,4,6,7,8\}$. Recall in Figure 1(b) $H_{0}$ 's clock leads $H_{1}$ 's clock by one beacon interval. From $H_{1}$ 's point of view, the quorum adopted by $H_{0}$ equals $\{8,0,1,2,5\}$, which, by definition, belongs to $C_{9}(\{0,1,2,3,6\})$ and still intersects with $H_{1}$ 's quorum $\{1,4,6,7,8\}$. The above definition serves as the basis for most quorum schemes used in existing QPS protocols $[4,5,8,16,19]$.

In the following, we generalize the definition of cyclic coterie to the notion of hyper quorum system. First, we define a useful notation:

Definition $3.4((n, m, l)$-revolving set) Given integers $n$, $m$, and $l$, where $0 \leq l \leq n-1$. Let $Q$ be a subset of
$U, U=\{0,1, \cdots, n-1\}$. We call $R_{n, m, l}(Q)$ an $(n, m, l)-$ revolving set of $Q$ if and only if $R_{n, m, l}(Q)=\{(q+i n)-l$ : $0 \leq(q+i n)-l \leq m-1, \forall q \in Q, i \in \mathbb{Z}\}$.

Intuitively, $R_{n, m, l}(Q)$ is a projection of $Q$ from the modulo$n$ plane onto the modulo- $m$ plane with an index shift $l$. For example, consider two quorums $Q_{0}=\{1,2,3\}$ and $Q_{1}=\{0,3,6,7,8\}$ shown in Figure 1(c), which are subsets of $U_{0}=\{0,1,2,3\}$ and $U_{1}=\{0,1, \cdots, 8\}$ respectively. Given two shift indices $l_{0}=2$ and $l_{1}=1$, we may project these two sets from the modulo- 4 and -9 planes onto the same modulo- 10 plane by using $R_{4,10,2}\left(Q_{0}\right)=$ $\{0,1,3,4,5,7,8,9\}$ and $R_{9,10,1}\left(Q_{1}\right)=\{2,5,6,7,8\}$ respectively. Note both $R_{4,10,2}\left(Q_{0}\right)$ and $R_{9,10,1}\left(Q_{1}\right)$ are subsets of a new universal set $U^{\prime}=\{0,1, \cdots, 9\}$. For convenience, we denote a group of revolving sets as $R_{n, m}(Q)=$ $\left\{R_{n, m, l}(Q): \forall l\right\}$.

Definition $3.5\left(\left(n_{0}, n_{1}, \cdots, n_{d-1} ; m\right)\right.$-hyper quorum system) Given integers $n_{0}, n_{1}, \cdots, n_{d-1}$ and $m$, where $d \in \mathbb{Z}$. Let $X=\left\{Q_{0}, Q_{1}, \cdots, Q_{d-1}\right\}$ be a set with the element $Q_{i}, 0 \leq i \leq d-1$, a nonempty subset of the universal set $U_{i}=\left\{0,1, \cdots, n_{i}-1\right\}$ over the modulo- $n_{i}$ plane. We call $X$ an $\left(n_{0}, n_{1}, \cdots, n_{d-1} ; m\right)$ hyper quorum system if and only if the set $R_{n_{0}, m}\left(Q_{0}\right) \cup R_{n_{1}, m}\left(Q_{1}\right) \cup \cdots \cup R_{n_{d-1}, m}\left(Q_{d-1}\right)$ forms an $m$-coterie.

Basically, a hyper quorum system guarantees the intersection between the projections of quorums over a plane, as depicted in Figure 2. Following the example shown in Figure 1 (c) where $Q_{0}=\{1,2,3\}$ and $Q_{1}=\{0,3,6,7,8\}$. Given a reference point of time, $t$. Suppose at time $t$ the stations $H_{0}$ and $H_{1}$ are in their $2^{\text {nd }}$ and $1^{\text {st }}$ beacon interval respectively. Then $H_{0}$ and $H_{1}$ are guaranteed to overlap in at least one awake beacon interval within the 10 beacon intervals after $t$, since $R_{4,10,2}\left(Q_{0}\right) \cap R_{9,10,1}\left(Q_{1}\right) \neq \varnothing$. Actually, we may easily verify that given any reference point of time where $H_{0}$ and $H_{1}$ are in their $l_{0}^{\text {th }}$ and $l_{1}^{\text {th }}$ beacon intervals respectively, $H_{0}$ and $H_{1}$ are guaranteed to overlap within 10 beacon intervals. Formally, we have $R_{4,10, l_{0}}\left(Q_{0}\right) \cap$ $R_{9,10, l_{1}}\left(Q_{1}\right) \neq \varnothing$ for all $l_{0}$ and $l_{1}, 0 \leq l_{0} \leq 3$ and $0 \leq l_{1} \leq 8$. Therefore, the set $\{\{1,2,3\},\{0,3,6,7,8\}\}$ is a $(4,9 ; 10)$-hyper quorum system. In Definition $3.5, X$ is said to be $d$-dimensional and the elements of $X$ are termed quorums.

Notice that a revolving set $R_{n, m, l}(Q)$ degenerates into a cyclic set $C_{n,(-l \bmod n)}(Q)$ when $m=n$. An $\left(n_{0}, n_{1}, \cdots, n_{d-1} ; m\right)$-hyper quorum system degenerates into a traditional $n$-cyclic quorum system when $n=n_{0}=$ $n_{1}=\cdots=n_{d-1}=m$.

By following the example given in Figure 2, we may observe that, first, HQS ensures a shift-invariant intersection, 
therefore supports any QPS protocol naturally. Suppose $H_{0}$ 's clock is shifted 1 beacon interval ahead. The projected quorum of $H_{0}$ at time $t$ becomes $R_{4,10,(2+1)}\left(Q_{0}\right)$, which, by definition, belongs to $R_{4,10}\left(Q_{0}\right)$ and still intersects with $R_{9,10,1}\left(Q_{1}\right)$. Second, starting from any reference point of time, $H_{0}$ and $H_{1}$ are able to exchange their beacon frames within $m$ beacon intervals. An $\left(n_{0}, n_{1}, \cdots, n_{d-1} ; m\right)$ hyper quorum system guarantees the worst-case neighbor discovery time $O(m) \bar{B}$. Note the FPP-based scheme [5] also gives an HQS. However, it does not guarantee a specific value of $m$ given $n_{0}$ to $n_{d-1}$. The neighbor discovery time is not predictable with this scheme.

It remains a challenging issue to efficiently construct an HQS given arbitrary modulo planes and arbitrary dimensions. Current schemes $[4,5]$ generate an HQS only when $n_{0}, n_{1}, \cdots, n_{d-1}$ are either squares or primes. Moreover, the HQS proposed in [5] is constructed by exhaustive searches. In the next section, we present algorithms that can systematically generate two different classes of HQS in $O(1)$ time over arbitrary modulo planes.

\subsection{Constructing Schemes for HQS}

We present two HQS constructing schemes that offer flexibility in supporting arbitrary values of $n$ and $d$ while keeping the quorum size close to the lower bound $\sqrt{n}$ [8].

Extended Grid (EG) scheme: Consider $d$ integers $n_{0}$, $n_{1}, \cdots, n_{d-1}$, where $n_{0}<n_{1}<\cdots<n_{d-1}$. Let $\phi_{i}=$ $\min \left\{\left\lfloor\sqrt{n_{i}}\right\rfloor,\left[\sqrt{\left(n_{d-1}+1\right) / 2}\right\rfloor\right\}$ and $q_{i}=\left\lfloor n_{i} / \phi_{i}\right\rfloor$ for all $i, 0 \leq i \leq d-1$. We define an extended grid quorum $G_{i}$ over the modulo- $n_{i}$ plane as follows:

$$
G_{i}=\left\{0,1, \cdots, \phi_{i}-1, g_{1}, g_{2}, \cdots, g_{q_{i}-1}\right\},
$$

where $\phi_{i}-1<g_{1} \leq 2 \phi_{i}-1$ and $0<g_{j+1}-g_{j} \leq$ $\phi_{i}$ for all $j, 1 \leq j \leq q_{i}-2$. Essentially, $G_{i}$ contains $\phi_{i}$ continuous elements from 0 to $\phi_{i}-1$, followed by $q_{i}-$ 1 interspaced elements with mutual distances less than or equal to $\phi_{i}$. Note with the above definition, $G_{i}$ is not unique.

The name of this scheme comes from the fact that when $n_{i}$ is a square and $g_{j+1}-g_{j}=\phi_{i}, G_{i}$ degenerates into a grid-based quorum. For example, let $d=2, n_{0}=9$ and $n_{1}=20$. By fixing $g_{j+1}-g_{j}=3$, we have $G_{0}=$ $\{0,1,2,5,8\}$, a quorum comprising a column and a row in the $3 \times 3$ grid.

It can be shown that $\left\{G_{0}, G_{1}, \ldots, G_{d-1}\right\}$ forms a $d$ dimensional HQS for some value of $m$. Instead of proving this, we will show that any pair of $G_{i}$ and $G_{j}$ forms a $\left(n_{i}, n_{j} ; m\right)$-hyper quorum system with a better bounded value of $m$. First, we define the heads of a revolving set $R_{n, m, l}(Q)$ to be those elements projected from the smallest element in $Q$ (note there could be none, or more than one head). In the previous example shown in Figure 2, the elements 3 and 7 are heads of $R_{4,10,2}\left(Q_{0}\right)$, and 8 is the head of $R_{9,10,1}\left(Q_{1}\right)$.
Theorem 3.1 Given $d$ integers $n_{0}, n_{1}, \cdots, n_{d-1}$, where $n_{0}<n_{1}<\cdots<n_{d-1}$. For any $i$ and $j, 0 \leq i \leq j \leq$ $d-1$, the set $\left\{G_{i}, G_{j}\right\}$ forms an $\left(n_{i}, n_{j} ; \phi_{i}+n_{j}-1\right)$-hyper quorum system.

Proof. Let $m=\phi_{i}+n_{j}-1$, we show that $R_{n_{i}, m, a}\left(G_{i}\right) \cap$ $R_{n_{j}, m, b}\left(G_{j}\right) \neq \varnothing$ for all $a$ and $b, 0 \leq a \leq n_{i}$ and $0 \leq b \leq n_{j}$. Recall that the heads of $R_{n_{j}, m, b}\left(G_{j}\right)$ are the elements projected from the first element 0 in $G_{j}$. Let $h$ be the first head in $R_{n_{j}, m, b}\left(G_{j}\right)$. Since $m \geq n_{j}$, h exists and $h \leq n_{j}-1$. If $h$ is included in $R_{n_{i}, m, a}\left(G_{i}\right)$, we finish the proof. Otherwise, consider two elements $s$ and $t$ in $R_{n_{i}, m, a}\left(G_{i}\right)$ such that $s<h<t$. By definition of $G_{i}$, any two interspaced elements in $R_{n_{i}, m, a}\left(G_{i}\right)$ must have mutual distance less than or equal to $\phi_{i}$. We have $t \leq s+\phi_{i}$, leading to $t \leq h+\phi_{i}-1 \leq \phi_{i}+n_{j}-2=m-1$. The element $t$ exists. On the other hand, by definition of $G_{j}, h$ is a head of $R_{n_{j}, m, b}\left(G_{j}\right)$ implies that there exist $\phi_{j}-1$ continuous elements after $h$ in $R_{n_{j}, m, b}\left(G_{j}\right)$. Since $t \leq h+\phi_{i}-1 \leq h+$ $\phi_{j}-1$, the element $t$ must also be included in $R_{n_{j}, m, b}\left(G_{j}\right)$. We have $R_{n_{i}, m, a}\left(D_{i}\right) \cap R_{n_{j}, m, b}\left(D_{j}\right) \neq \varnothing$.

Difference Set (DS) scheme: Consider $d$ integers $n_{0}$, $n_{1}, \cdots, n_{d-1}$, where $n_{0}<n_{1}<\cdots<n_{d-1}$. Let $\phi=$ $\left\lceil\sqrt{\left(n_{d-1}+1\right) / 2}\right\rceil$ and $q_{i}=\left\lceil\frac{n_{i}+1}{2 \phi}\right\rceil$ for all $i, 0 \leq i \leq$ $d-1$. We define another quorum $D_{i}$ over the modulo- $n_{i}$ plane as follows:

$$
D_{i}=\left\{0,1, \cdots, \phi-1, d_{1}, d_{2}, \cdots, d_{q_{i}-1}\right\},
$$

where $\phi-1<d_{1} \leq 2 \phi-1,0<d_{j+1}-d_{j} \leq \phi$ for all $j, 1 \leq j \leq q_{i}-2$, and $d_{q_{i}-1} \geq\left(n_{i}-1\right) / 2$. Basically, $D_{i}$ contains $\phi$ continuous elements from 0 to $\phi-1$, followed by $q_{i}-1$ interspaced elements with mutual distances less than or equal to $\phi$. Note, again, $D_{i}$ is not unique with the above definition. Note $D_{i}$ is a difference set [14].

Theorem 3.2 Given $d$ integers $n_{0}, n_{1}, \cdots, n_{d-1}$, where $n_{0}<n_{1}<\ldots<n_{d-1}$. Let $\phi=\left\lceil\sqrt{\left(n_{d-1}+1\right) / 2}\right\rceil$. For any $i$ and $j, 0 \leq i \leq j \leq d-1$, the set $\left\{D_{i}, D_{j}\right\}$ forms an $\left(n_{i}, n_{j} ;\left\lfloor\left(n_{i}-1\right) / 2\right\rfloor+n_{j}+\phi-1\right)$-hyper quorum system. Proof. Let $m=\left\lfloor\left(n_{i}-1\right) / 2\right\rfloor+n_{j}+\phi-1$, we show that $R_{n_{i}, m, a}\left(D_{i}\right) \cap R_{n_{j}, m, b}\left(D_{j}\right) \neq \varnothing$ for all $a$ and $b, 0 \leq a \leq$ $n_{i}$ and $0 \leq b \leq n_{j}$. Let $h$ be the first head in $R_{n_{j}, m, b}\left(D_{j}\right)$. Since $m \geq n_{j}$, the element $h$ exists and $h \leq n_{j}-1$. If $h$ is included in $R_{n_{i}, m, a}\left(D_{i}\right)$, we finish the proof. Otherwise, consider two elements $s$ and $t$ in $R_{n_{i}, m, a}\left(D_{i}\right)$ such that $s<$ $h<t$.

Case 1: If $t \leq s+\phi$, we have $t \leq h+\phi-1 \leq m-$ 1. The element $t$ exists. On the other hand, by definition of $D_{j}, h$ is a head of $R_{n_{j}, m, b}\left(D_{j}\right)$ implies that there exist $\phi-1$ continuous elements after $h$ in $R_{n_{j}, m, b}\left(D_{j}\right)$. The element $t$ must also be included in $R_{n_{j}, m, b}\left(D_{j}\right)$. We have 
$R_{n_{i}, m, a}\left(D_{i}\right) \cap R_{n_{j}, m, b}\left(D_{j}\right) \neq \varnothing$.

Case 2: Ift $>s+\phi$, by definition of $D_{i}$, the element t must be a head of $R_{n_{i}, m, a}\left(D_{i}\right)$. There exist $\phi-1$ continuous elements after $t$ in $R_{n_{i}, m, a}\left(D_{i}\right)$. Note the element $t$ and all $\phi-1$ continuous elements after $t$ must be included in $R_{n_{i}, m, a}\left(D_{i}\right)$, since $d_{q_{i}-1} \geq\left(n_{i}-1\right) / 2$ implies that $t \leq$ $s+\left(n_{i}-\left(n_{i}-1\right) / 2\right)=s+\left(n_{i}+1\right) / 2 \leq h+\left(n_{i}+1\right) / 2-$ $1=h+\left(n_{i}-1\right) / 2$, leading to $t \leq h+\left\lfloor\left(n_{i}-1\right) / 2\right\rfloor \leq$ $\left\lfloor\left(n_{i}-1\right) / 2\right\rfloor+n_{j}-1=m-\phi$, and therefore $t+\phi-$ $1 \leq m-1$. On the other hand, all elements between $h$ and $t+\phi-1$ in $R_{n_{j}, m, b}\left(D_{j}\right)$ must have mutual distances less than or equal to $\phi$, since $h+d_{q_{j}-1} \geq h+\left(n_{j}-1\right) / 2 \geq$ $h+\left(n_{i}-1\right) / 2 \geq t$. By pigeon hole principle there must exist at least one element between $t$ and $t+\phi-1$ in $R_{n_{j}, m, b}\left(D_{j}\right)$. Hence, $R_{n_{i}, m, a}\left(D_{i}\right) \cap R_{n_{j}, m, b}\left(D_{j}\right) \neq \varnothing$.

The HQS-based Power Saving (HQPS) protocol inherits the design (e.g., beacon frames, neighbor maintenance, data transmission procedure, etc.) of a traditional QPS protocol, as we have seen in Section 2.

\section{Performance Evaluation}

In this section, we evaluate the performance of HQPS by taking both the theoretical analysis and simulation results. We implement our simulation based on the $n s-2$ simulator [1] with CMU wireless extension. The simulation is conducted in a $500 \times 500 \mathrm{~m}^{2}$ static network with 50 randomly distributed stations. Each station has half-duplex wireless channel of rate $2 \mathrm{Mbps}$ and transmission range 100 meters. We set the duration of beacon interval and ATIM window 100 and $25 \mathrm{~ms}$ respectively. The mean packet size is 256 bytes and each station is supplied with the Poisson traffic [18] with rate varying from 2 to $t_{\max } \mathrm{Kbps}$, where $t_{\max }=40$ by default. The power consumption rates of the wireless module are set $1650,1400,1150$, and $45 \mathrm{~mW}$ in transmit, receive, idle, and sleep modes respectively [10]. All stations are synchronous in their clocks.

To evaluate the performance of a quorum scheme, we define a theoretical metric - quorum ratio, which denotes the proportion of beacon intervals in a cycle where a station is required to awake. Specifically, it is defined as $|Q| / n$, where $|Q|$ is the quorum size and $n$ is the cycle length.

We compare with the previous studies AQEC [4] and AAPM [5]. These two protocols adopt Grid and FPP as the underlying quorum schemes respectively. To ensure valid neighbor maintenance in routing protocols [15], the cycle length $n$ is considered to be less than or equal to $n_{\max }=25$. We set $\phi=4$. All schemes adopt the rule-based adaptation criteria [4] to dynamically adjust $n$. In our experiments, the

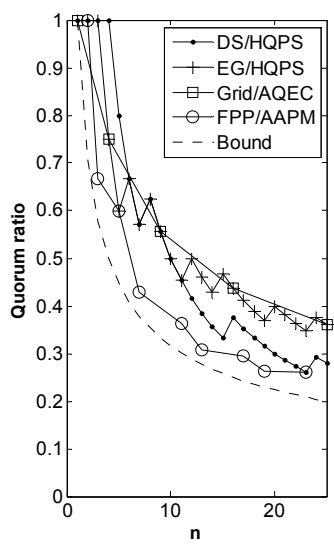

(a)

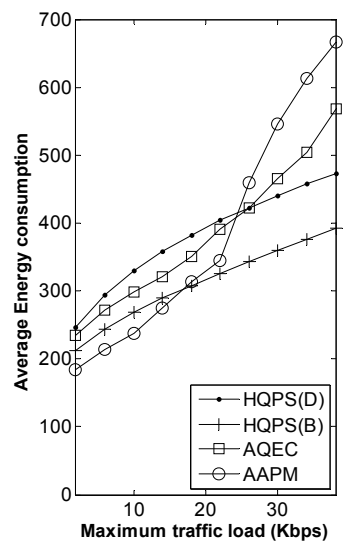

(b)
Figure 3. Quorum ratio and average energy consumption rate.

rules for $\mathrm{AQEC}$ are:

\begin{tabular}{cl}
\hline Grid size $(\boldsymbol{n})$ & \multicolumn{1}{c}{ Used when } \\
\hline $1 \times 1(1)$ & traffic $\geq t_{\max } \cdot(1 / 1)$ \\
$2 \times 2(4)$ & $t_{\max } \cdot(1 / 1)>$ traffic $\geq t_{\max } \cdot(3 / 4)$ \\
$3 \times 3(9)$ & $t_{\max } \cdot(3 / 4)>$ traffic $\geq t_{\max } \cdot(5 / 9)$ \\
$4 \times 4(16)$ & $t_{\max } \cdot(5 / 9)>$ traffic $\geq t_{\max } \cdot(7 / 16)$ \\
$5 \times 5(25)$ & $t_{\max } \cdot(7 / 16)>$ traffic \\
\hline
\end{tabular}

AAPM follows the similar similar rules that corresponds to its offered quorum ratios. HQPS, on the other hand, employs an analytic model $[13,18,20]$ to pick a proper scheme and $n$. We omit the details of the model due to the space limitation. Note that to give a fair comparison, all protocols employ the same design of cycle pattern as described in Section 2.

\subsection{Theoretical Analysis: the Quorum Ratio}

We first explore the quorum ratio of different schemes by varying the cycle length $n$ from 1 to $n_{\max }$. As shown in Figure 3(a), all schemes give smaller quorum ratios as $n$ increases. In particular, the ratio of FPP approaches the theoretical bound $1 / \sqrt{n}$ [8]. This ratio is shown to be optimal [5]. However, the optimum in quorum size does not necessary imply the optimum in performance. Notice that FPP and Grid cannot produce quorums given arbitrary cycle lengths. This leads to a sparse configuration density. During the rule-based adaptation, $n$ may shrink very fast when there is only little increment in traffic load. A station may tend to remain awake more than necessary. Note the quorum ratios of Grid and EG overlap when $n$ is a square. This is 
simply because EG is a generalization of the Grid scheme.

Next, we evaluate the performance of different protocols based on the simulation results.

\subsection{Energy Conservation}

In this section, we evaluate the energy efficiency of all protocols under different loads. We vary $f_{\max }$ from 2 to 40 Kbps. The experimental results are shown in Figure 3(b), where HQPS(D) and HQPS(B) demonstrate the behavior of HQPS given delay-constrained and best-effort traffic respectively. As we can see, all protocols give higher energy consumption rate as $f_{\text {max }}$ increases. This is because of the frequent ATIM notification and data transmission procedures. Notice that the energy consumption rates of AQEC and AAPM grow significantly when $f_{\max }>20 \mathrm{Kbps}$. This is mainly due to the sparse configuration density, as we have seen in Figure 3(a). When the offered load is close to the maximum support load, $n$ shrinks very fast and keeps a station awake most of time. On the other hand, HQPS give relatively stable energy consumption rate under all loads. As compared with AQEC and AAPM, HQPS offers competitive energy efficiency under light traffic loads; while giving respectively up to $31 \%$ and $41 \%$ reduction in energy consumption rate under heavy loads.

\section{Conclusions}

In this paper, we generalized traditional quorum systems and proposed the Hyper Quorum System (HQS). We showed that HQS is fully adaptive in the sense that a station can select any value of cycle length that is best suited to its own requirements in terms of packet delay and power constraint. Two HQS constructing schemes were presented that facilitate power saving under both delay-constrained and best-effort traffic. Experimental results showed that our HQS-based power management protocol renders significantly more stable performance under various traffic loads as compared with traditional QPS protocols. In particular, it gives up to $41 \%$ improvement in energy efficiency under heavy traffic loads.

\section{References}

[1] The network simulator (ns-2). http://www.isi.edu/nsnam/ns.

[2] M. Anand, E.B. Nightingale, and J. Flinn. Self-tuning wireless network power management. Wireless Networks, 11(4):451-469, 2005.

[3] P.A. Bernstein, V. Hadzilacos, and N. Goodman. Concurrency Control and Recovery in Database Systems. AddisonWesley, 1987.

[4] C.M. Chao, J.P. Sheu, and I.C. Chou. An adaptive quorumbased energy conserving protocol for ieee 802.11 ad hoc netowrks. IEEE Transactions on Mobile Computing, 5(5):560 570, 2006.
[5] Z.T. Chou. Optimal adaptive power management protocols for asynchronous wireless ad hoc networks. In Proc. of WCNC, pages 61-65, 2007.

[6] IEEE 802 LAN/MAN Standards Committee. Wireless LAN Medium Access Control and Physical Layer Specifications, 1999.

[7] L.M. Feeney and M. Nilsson. Investigating the energy consumption of a wireless network interface in an ad hoc networking environment. In Proc. of INFOCOM, pages 1548$1557,2001$.

[8] J.R. Jiang, Y.C. Tseng, C.S. Hsu, and T.H. Lai. Quorumbased asynchronous power-saving protocols for ieee 802.11 ad hoc networks. Mobile Networks and Applications, 10(12):169-181, 2005.

[9] E.S. Jung and N. Vaidya. An energy efficient mac protocol for wireless lans. In Proc. of INFOCOM, pages 1756-1764, 2002.

[10] E.S. Jung and N.H. Vaidya. An energy efficient mac protocol for wireless lans. In Proc. of INFOCOM, 2002.

[11] R. Krashinsky and H. Balakrishnan. Minimizing energy for wireless web access with bounded slowdown. Wireless Networks, 11(1-2):135-148, 2005.

[12] R. Kravets and P. Krishnan. Application-driven power management for mobile communication. Wireless Networks, 6(4):263-277, 2000.

[13] D. Logothetis, V. Mainkar, and K. Trivedi. Transient analysis of non-markovian queues via markov regenerative processes. Probability and Statistics: AJ Medhi Festschrift, 1996.

[14] W.S. Luk and T.T. Wong. Two new quorum based algorithms for distributed mutual exclusion. In Proc. of ICDCS, pages 100-106, 1997.

[15] C.E. Perkins and E.M. Royer. Ad-hoc on-demand distance vector routing. In Proc. of WMCSA, 1999.

[16] Y.C. Tseng, C.S. Hsu, and T.Y. Hsieh. Power-saving protocols for ieee 802.11-based multi-hop ad hoc networks. In Proc. of INFOCOM, pages 200-209, 2002.

[17] W. Ye, J. Heidemann, and D. Estrin. Medium access control with coordinated adaptive sleeping for wireless sensor networks. IEEE/ACM Transactions on Networking, 12(3):493506, 2004.

[18] R. Zheng. Design, analysis, and empirical evaluation of power management in multihop wireless networks. Technical Report UIUCDCS-R-2003-2381, 2003.

[19] R. Zheng, J.C. Hou, and L. Sha. Optimal block design for asynchronous wake-up schedules and its applications in multihop wireless networks. IEEE Transactions on Mobile Computing, 5(9):1228-1241, 2006.

[20] R. Zheng, J.C. Hou, and L. Sha. Performance analysis of power management policies in wireless networks. IEEE Transactions on Wireless Communications, 5(6):1351-1361, 2006.

[21] R. Zheng and R. Kravets. On-demand power management for ad hoc networks. In Proc. of INFOCOM, pages 481-491, 2003. 